\title{
Über die Struktur der Heitz-Leyonschen Kristalle*
}

\author{
Von Wilhelm Menke \\ Aus dem Botanischen Institut der Universität zu Köln \\ (Z. Naturforschg. 17 b, 188-190 [1962] ; eingegangen am 18. Oktober 1961)
}

\begin{abstract}
Die He it z-L e y o n schen Kristalle der Plastiden von Chlorophytum comosum bestehen aus spiralig gewundenen Tubuli, die in einer Matrix eingebettet sind. Die Anordnung der spiraligen Bauelemente ist wahrscheinlich eine hexagonale. Die Windungen benachbarter Spiralen sind gegeneinander ausgerichtet. Daher besitzen Heitz-Leyon sche Kristalle einen dreidimensionalen Ordnungszustand.
\end{abstract}

Die Heitz-Leyon schen Kristalle der squamulae intravaginales von Elodea canadensis bestehen, wie kürzlich gefunden wurde, aus Tubuli in hexagonal dichtester Anordnung ${ }^{1}$. Bei anderen Pflanzen besitzen diese Plastideneinschlüsse nach den von Heitz ${ }^{2}$, Leyon ${ }^{3}$ und anderen Autoren ${ }^{4}$ veröffentlichten elektronenmikroskopischen Aufnahmen einen abweichenden Feinbau. Daher mußte untersucht werden, ob es sich bei den kristallinen Plastideneinschlüssen überhaupt um homologe Strukturen handelt ${ }^{5}$.

$\mathrm{Zu}$ diesem Zweck war es notwendig, den morphologischen Bau authentischer Heitz-Le y o n scher Kristalle aufzuklären. Als Untersuchungsobjekt wurde Chlorophytum comosum $^{6}$ gewählt, weil Heitz bei dieser Pflanze die kristallinen Plastidenzentren entdeckte.

Nach den in der vorliegenden Arbeit wiedergegebenen Aufnahmen zeigen Schnitte durch kristalline Plastidenzentren von Chlorophytum ein recht verschiedenes Aussehen und nur einige Bilder (z. B. Abb. $6^{* *}$ und 7) sind mit Aufnahmen der oben genannten Autoren vergleichbar. Da Grund zu der Annahme bestand, daß den verschiedenen Aufnahmen ein und dieselbe Kristallstruktur zugrunde liegt, mußte ein räumliches Modell gesucht werden, das unter verschiedenen Winkeln geschnitten, ent-

* Herrn Professor Dr. Emil Heitz zum 69. Geburtstag gewidmet.

1 W. Menke, Z. Naturforschg. 15 b, 800 [1960].

2 E. Heitz, Exp. Cell. Res. 7, 606 [1954], Experientia [Basel] 12, 476 [1956]; 16, 265 [1960].

3 H. Leyon, Exp. Cell. Res. 7, 609 [1954].

4 E. Perner, Z. Naturforschg. 11 b, 560 [1956]; D. v. Wett Stern, Brookhaven Symposia in Biology (Upton/N. Y.) 11, 138 [1958]; D. v. Wettstein, in: D. Rudnick, Developmental Cytology 123. The Roland Press Company, 1959; K. Mühlethaler u. A. Frey-Wyssling, J. Biophys. Biochem. Cytol. 6, 507 [1959];S. Murakami, J. Electronmicroscopy 9, 91 [1960] ; S. KleiN, J. Biophys. Biochem. Cytol. 8, 529 [1960]; P. Sitte, Protoplasma 53, 438 [1961]. sprechende Strukturen ergibt, wie sie die elektronenmikroskopischen Aufnahmen zeigen.

Aus den in Abb. 1 und 2 wiedergegebenen Aufnahmen geht hervor, daß die Heitz-Leyon schen Kristalle von Chlorophytum aus spiralig gewundenen Tubuli aufgebaut sind. Eine andere Deutung der zickzack-förmigen Gebilde dürfte kaum möglich sein, da sie an den Ecken ringförmige optische Querschnitte der Tubuliwände erkennen lassen. Der Durchmesser der Tubuli beträgt etwa $160 \AA$, der Durchmesser der Spiralen etwa $450 \AA$. Einheiten. Die Winkel zwischen den Schenkeln der zickzack-förmigen Bänder schwanken um $64^{\circ}$, so daß die Spiralen eine Steigung von etwa $32^{\circ}$ besitzen. Sie sind gleich weit voneinander entfernt und anscheinend in eine Matrix eingebettet. Bemerkenswert ist, daß die Windungen der Spiralen auf zwei Scharen von parallelen Geraden liegen. Diese besitzen den gleichen Abstand voneinander und schneiden sich unter dem doppelten Steigungswinkel, also unter etwa 64 Grad.

Der in Abb. 6 wiedergegebenen Aufnahme liegt scheinbar eine andere Objektstruktur zugrunde. Diese Aufnahme stammt von einem dickeren Schnitt. Für die Deutung ist wichtig, daß sich durch die netzartige Struktur zwanglos zwei Scharen von parallelen Geraden legen lassen, die sich unter dem

5 E. Schnepf (Protoplasma 54, 310 [1961]) bezweifelt, daß die kristallinen Plastideneinschlüsse in den squamulae intravaginales von Elodea echte $\mathrm{H}$ e i t z - L e y o n sche Kristalle sind. Ich danke Herrn Dr. Schnepf, daß er mir seine Arbeit schon als Manuskript zuschickte.

${ }^{6}$ Schnitte aus dem unteren Drittel eines $6 \mathrm{~cm}$ langen Blattes. Variegate Form, deren Blätter in schwachem Licht angezogen, gleichmäßig grün, bei starker Beleuchtung jedoch gestreift sind. Fixierung: Kaliumpermanganat; Einbettung: Polymethacrylat; Schnitte: Eigenbaumikrotom; Aufnahmen: Siemens Elmiskop I.

** Abb. 1, 2, 3, 5-8 s. Tafel S. 190 a und b. 
eben charakterisierten Winkel von etwa $64^{\circ}$ schneiden. Modellversuche ${ }^{7}$ ergaben, daß ein entsprechendes Bild entsteht, wenn spiralig gewundene Tubuli, deren Windungen in der angegebenen Weise zueinander ausgerichtet sind, in der in Abb. 4 a gezeichneten Anordnung im Schnitt liegen. Die Spiralen überdecken sich teilweise; denn die ringförmigen Querschnitte an den Ecken erscheinen einfach. Aufnahmen mit scheinbar netzförmigen Strukturen wurden übrigens am häufigsten gefunden. Da man nun annehmen darf, daß sich die in Abb. 4 a angegebene Anordnung der Spiralen im Kristall wiederholt, so erhält man durch Translatation in Richtung senkrecht zur Schnittebene die gesuchte Kristallstruktur. Das Ergebnis der Translatation ist eine Anordnung, bei der jede Spirale von sechs anderen gleich weit entfernten Spiralen umgeben ist, also ein hexagonales Gitter (Abb. $4 \mathrm{~b})$.

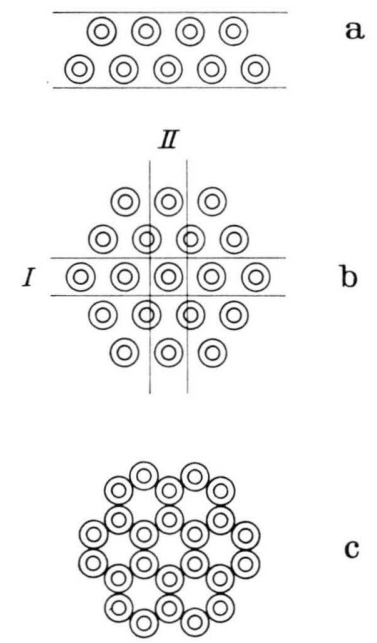

Abb. 4. Schema zur Kristallstruktur der H e i t z-L e y o n schen Kristalle. In Richtung der Spiralenachsen gesehen, erscheinen die Spiralen ringförmig. Die in a angegebene Anordnung im Schnitt ergibt Bilder, die Abb. 6 entsprechen; b das aus a abgeleitete hexagonale Strukturmodell; c Strukturmodell, das ebenfalls mit einem Teil der Aufnahmen vereinbar ist.

Es muß nun untersucht werden, inwieweit die auf den verschiedenen elektronenmikroskopischen Aufnahmen abgebildeten Strukturen der eben abgeleiteten hexagonalen Kristallstruktur im einzelnen entsprechen. Dabei ist es zweckmäßig, von zwei Schnitt-

\footnotetext{
7 Aus Trovidur hergestellte Spiralen wurden in Plexiglas eingebettet und in verschiedenen Richtungen und unter verschiedenen Winkeln in Scheiben zerlegt. Herrn Feinmechanikermeister A. Wilhelm danke ich für die Herstellung der Modelle.
}

richtungen auszugehen, die in Abb. $4 \mathrm{~b}$ angedeutet und mit I und II bezeichnet sind. Schnittrichtung I verläuft parallel zu den Flächen des hexagonalen Prismas und parallel zu den Spiralenachsen. Schnitte von der Dicke einer Spirale, bei denen die Spiralen nicht angeschnitten sind, zeigen parallel zueinander verlaufende Spiralen (Abb. 3 und 8), deren Abstand, gemessen von Spiralenachse zu Spiralenachse, mit a bezeichnet werden soll. Dickere Schnitte, die zwei hintereinanderliegende Reihen von Spiralen enthalten, geben das schon besprochene netzartig erscheinende Strukturbild. Wenn eine Reihe von Spiralen angeschnitten ist, so führt das zu Unregelmäßigkeiten, wie sie in Abb. 6 an einigen Stellen zu erkennen sind. Wenn die beiden Flächen eines Schnittes zufällig durch die Achsen der hintereinanderliegenden Reihen von Spiralen verlaufen, der Schnitt also zwei halbe Reihen von Spiralen enthält, so ergänzen sich die Abschnitte der angeschnittenen Spiralen im Bilde zu vollständigen Spiralen. Diese verlaufen aber senkrecht zur ursprünglichen Spiralenrichtung (Abb. 3). Sind Schnitte, die etwa die Dicke eines Spiralendurchmessers besitzen, gegen die Spiralenachse um einen nicht zu großen Winkel geneigt, so entstehen Bilder, die den in Abb. 1 und 2 wiedergegebenen entsprechen.

Schnittrichtung II verläuft senkrecht zu den Flächen des hexagonalen Prismas (Abb. 4 b). Aufnahmen von dicken Schnitten zeigen parallel zueinander verlaufende Spiralen, deren scheinbarer Abstand jedoch kleiner ist als der in Schnittrichtung I gefundene. Er beträgt $a / 2 \sqrt{3}$. Ein Beispiel für diesen Fall gibt Abb. 5, wobei die Schnittrichtung freilich nicht genau parallel zu den Spiralenachsen verläuft.

Außer den hier wiedergegebenen wurde noch eine Reihe anderer Strukturmuster von $\mathrm{Heitz}$ Leyouschen Kristallen erhalten (z. B. Abb. 8 links). Diese sind schwer zu deuten, da beliebig orientierte Schnitte verschiedener Dicke zu einer Fülle verschiedener Bilder führen. Obwohl zur Zeit mehr als 900 Aufnahmen von Heitz-Leyonschen Kristallen zur Auswertung vorliegen, wurde nie ein Schnitt gefunden, in dem die Spiralen senkrecht getroffen sind. Ein solcher Schnitt würde die Richtigkeit der hier abgeleiteten hexagonalen Anordnung der spiraligen Strukturelemente entweder endgültig beweisen oder zur Auffindung der wirklichen Struktur führen. Außer dem beschriebenen hexagonalen wurde noch eine Reihe anderer, ähnlicher Modelle geprüft. Diese führten jedoch zu weni- 
ger befriedigenden Ergebnissen. So kann man von einer Anordnung der Spiralen, wie sie in Abb. $4 \mathrm{c}$ angegeben ist, ebenfalls Bilder wie Abb. 1, 2 und 6, nicht aber wie Abb. 3 und 5 erhalten. Die anderen Modelle waren mit den gefundenen Strukturen überhaupt nicht vereinbar. Daher ist zur Zeit das vorgeschlagene Modell als das wahrscheinlichste anzusehen. Es soll jedoch nicht verschwiegen werden, daß sich einige elektronenmikroskopische Aufnahmen nicht mit dem hexagonalen Modell erklären ließen. In diesen Fällen liegen den Aufnahmen anscheinend andere Anordnungen der Spiralen zugrunde, die vielleicht als Übergangsstrukturen angesehen werden dürfen.

Abschließend sei noch auf die Frage eingegangen, ob es sich bei den kristallinen Plastidenzentren um homologe Bildungen handelt. Den He i t z - Le y o n -

8 W. Menke, Experientia [Basel] 16, 537 [1960]. schen Kristallen von Chlorophytum, die wahrscheinlich einen weit verbreiteten Typ darstellen, und den Kristallen von Elodea ist zweierlei gemeinsam: Die Bauelemente der Kristallstruktur sind in beiden Fällen Tubuli. Ferner ist die Anordnung der Tubuli in beiden Fällen eine hexagonale. Wenn sich nachweisen ließe, daß die Tubuli in beiden Fällen aus der inneren Plastidenmembran entstehen, so wäre der Beweis für die Gleichwertigkeit dieser Gebilde erbracht. Erwähnt sei noch, daß bei Chlorophytum auch amorphe Plastidenzentren vorkommen, und daß das Lamellarsystem der Chloroplasten von Chlorophytum, wie aus Abb. 1, 3 und 8 zu ersehen ist, dem kürzlich aufgeklärten allgemeinen Bauprinzip entspricht.

Die Untersuchungen wurden mit Unterstützung der Deutschen Forschungsgemeinschaft ausgeführt. Fräulein BARBARA Fricke danke ich für ihre gewissenhafte Mitarbeit.

\title{
Quantitative Bausteinanalyse der Stützmembran in der Zellwand von Escherichia coli B
}

\author{
Von H. H. Martin und H. Frank \\ Aus dem Max-Planck-Institut für Biologie, Abt. WEIDEL, Tübingen \\ (Z. Naturforschg. 17 b, 190-196 [1962] ; eingegangen am 30. Oktober 1961)
}

\begin{abstract}
The three concentric layers of the cell wall of E. coli B (the outer lipoprotein layer, the intermediate lipopolysaccharide layer and the inner protein- and mucopolymer containing rigid layer) contribute $60 \%, 12 \%$ and $21 \%$, respectively, to the total weight of the cell wall. - Treatment of the rigid layer with proteolytic enzymes removes the bulk of its protein components but leaves it otherwise intact: still rigid and cell-shaped, it is found to contain glucose, lipid and the mucopeptide constituents muramic acid, glucosamine, diaminopimelic acid, glutamic acid and alanine in molar ratios corresponding to the chemical compositions of known enzymatic mucopolymer split-products from E. coli-walls. Quantitative balance sheets of these components are made up and their implications on the structural concept of the rigid layer are discussed.
\end{abstract}

Die komplexe Zellwand von E. coliB verdankt ihre mechanische Festigkeit einer besonderen Zellwandschicht, wie kürzlich von WeIDEL und Mitarbb. ${ }^{1}$ gezeigt wurde. Diese Schicht (Stützmembran *, engl. rigid layer) kann präparativ gewonnen werden und erweist sich als allseitig geschlossener, länglicher, durch covalente Bindungen zusammengehaltener Beutel oder Schlauch von den Dimensionen der Colizelle.

Lysozym zerlegt das schlauchförmige Riesenmolekül in morphologisch übersichtlicher Weise: es zer-

1 W. Weidel, H. Frank u. H. H. Martin, J. gen. Microbiol. 22, 158 [1960].

* Wir ersetzen die früher ${ }^{3,7}$ verwendete Bezeichnung „Basalmembran" auf Vorschlag von W. WeIDEL durch den die fällt vollkommen in kleine, vorwiegend aus Protein bestehende Kügelchen, die sich als typisches Oberflächenmuster schon in der intakten Struktur elektronenmikroskopisch bemerkbar machen. Das Enzymsubstrat ist ein die Kügelchen zusammenhaltendes Mucopolymer. Es wird durch Sprengung glykosidischer Bindungen von Lysozym in seine Bauelemente zerlegt, die als Mucopeptide zu bezeichnen und in einer anderen Arbeit von WeIDel und Mitarbb. ${ }^{2}$ chemisch charakterisiert worden sind.

Die Vernetzung von Proteinkügelchen durch

Funktion der „rigid layer" besser beschreibenden Ausdruck „Stützmembran“.

2 J. Primosigh, D. Maass, H. Pelzer u. W. Weidel, Biochim. biophysica Acta 46, 68 [1961]. 